Applied Mathematical Sciences, Vol. 9, 2015, no. 11, 515 - 532
HIKARI Ltd, www.m-hikari.com
http://dx.doi.org/10.12988/ams.2015.411962

\title{
Hesitant Fuzzy Prefilters and Filters of EQ-algebras
}

\author{
Young Bae Jun \\ Department of Mathematics Education \\ Gyeongsang National University \\ Jinju 660-701, Korea \\ Seok-Zun Song* \\ Department of Mathematics \\ Jeju National University \\ Jeju 690-756, Korea
}

Copyright (c) 2014 Young Bae Jun and Seok-Zun Song. This is an open access article distributed under the Creative Commons Attribution License, which permits unrestricted use, distribution, and reproduction in any medium, provided the original work is properly cited.

\begin{abstract}
Application of hesitant fuzzy sets to EQ-algebras is discussed. The notions of hesitant fuzzy prefilters (filters) and positive implicative hesitant fuzzy prefilters (filters) of EQ-algebras are introduced, and several properties are investigated. Characterizations of hesitant fuzzy prefilters (filters) and positive implicative hesitant fuzzy prefilters (filters) are considered, and conditions for a hesitant fuzzy filter to be a positive implicative hesitant fuzzy filter are investigated. Finally, the extension property for a positive implicative hesitant fuzzy filter is established.
\end{abstract}

Mathematics Subject Classification: 03G25, 08A72, 08B05, 08B99

Keywords: (commutative, residuated) EQ-algebra, (positive implicative) prefilter, (positive implicative) filter, hesitant fuzzy prefilter (filter), positive implicative hesitant fuzzy prefilter (filter)

*Corresponding author 


\section{Introduction}

Algebras including EQ-algebras have played an important role in recent years and have its comprehensive applications in many aspects including dynamical systems and genetic code of biology (see [1], [4], [5], and [14]). Starting from the four DNA bases order in the Boolean lattice, Sáanchez et al. [12] proposed a novel Lie Algebra of the genetic code which shows strong connections among algebraic relationship, codon assignments and physicochemical properties of amino acids. Tian [13] introduced an important algebra, so called evolution algebras, and proposed applications in a lot of aspects such as in Non-Mendelian inheritance.

V. Novák and B. De Baets [9] introduced a new class of algebras, called EQalgebras, which are a natural algebra proposed as an algebra of truth values on the basis of which the fuzzy type theory (a higher-order fuzzy logic) should be developed. An EQ-algebra has three binary operations: meet $(\wedge)$, multiplication $(\otimes)$, and fuzzy equality $(\sim)$, and a unit element, whereas the implication $(\rightarrow)$ is derived from the fuzzy equality $(\sim)$. Filter theory plays a vital role in studying several algebraic structures such as residuated lattices, $M V$-algebras, $B L$-algebras, $R_{0}$-algebras, $M T L$-algebras, $B C K / B C I$-algebras, lattice implication algebras, and so forth. M. El-Zekey et al. [3] continued the study of EQ-algebras. They introduced and studied the prefilters and filters of EQalgebras. Liu and Zhang [6] discussed the implicative and positive implicative prefilters (filters) of EQ-algebras.

The hesitant fuzzy set which is introduced by Torra [15] is a useful generalization of the fuzzy set that is designed for situations in which it is difficult to determine the membership of an element to a set owing to ambiguity between a few different values. The hesitant fuzzy set permits the membership degree of an element to a set to be represented by a set of possible values between 0 and 1 (see [15] and [16]). The hesitant fuzzy set therefore provides a more accurate representation of people's hesitancy in stating their preferences over objects than the fuzzy set or its classical extensions. Hesitant fuzzy set theory has been applied to several practical problems, primarily in the area of decision making (see [11], [16] [18], [19], [20], [21], [22]).

In this paper, we apply the notion of hesitant fuzzy sets to EQ-algebras. We introduce the concepts of hesitant fuzzy prefilters (filters) and positive implicative hesitant fuzzy prefilters (filters) of EQ-algebras, and investigate several properties. We discuss their characterizations, and provide conditions for a hesitant fuzzy filter to be a positive implicative hesitant fuzzy filter. We establish the extension property for a positive implicative hesitant fuzzy filter. 


\section{Preliminaries}

We display basic definitions and properties of EQ-algebras that will be used in this paper. For more details of EQ-algebras, we refer the reader to [2], [3], $[6],[7],[8]$, and [10].

Definition 2.1 An EQ-algebra is an algebra $\mathcal{L}:=(L, \wedge, \otimes, \sim, 1)$ of type $(2,2,2,0)$ in which the following axioms are valid:

(L1) $(L, \wedge, 1)$ is a commutative idempotent monoid (i.e. $\wedge$-semilattice with top element 1$)$,

(L2) $(L, \otimes, 1)$ is a monoid and $\otimes$ is isotone with respect to $\leq$ (with $x \leq y$ defined as $x \wedge y=x)$,

(L3) $x \sim x=1$,

(L4) $((x \wedge y) \sim z) \otimes(a \sim x) \leq z \sim(a \wedge y)$,

$(\mathrm{L} 5)(x \sim y) \otimes(a \sim b) \leq(x \sim a) \sim(y \sim b)$,

(L6) $(x \wedge y \wedge z) \sim x \leq(x \wedge y) \sim x$

(L7) $x \otimes y \leq x \sim y$

for all $x, y, z, a, b \in L$.

The operation " $\wedge$ " is called meet (infimum) and " $\otimes$ " is called multiplication. If the multiplication is commutative in an EQ-algebra $\mathcal{L}$, then we say that $\mathcal{L}$ is a commutative EQ-algebra.

Let $\mathcal{L}$ be an EQ-algebra. For all $x \in L$, we put $\tilde{x}=x \sim 1$. We also define the derived operation, so called implication and denoted by $\rightarrow$, as follows:

$$
(\forall x, y \in L)(x \rightarrow y=(x \wedge y) \sim x) .
$$

An EQ-algebra $\mathcal{L}$ is said to be residuated if $(x \otimes y) \wedge z=x \otimes y$ if and only if $x \wedge((y \wedge z) \sim y)=x$ for all $x, y, z \in L$.

Proposition 2.2 Every (commutative) EQ-algebra $\mathcal{L}$ satisfies the following conditions for all $a, b, c, d \in L$ :

(1) If $a \leq b$, then $a \rightarrow b=1, a \sim b=b \rightarrow a, \tilde{a} \leq \tilde{b}, c \rightarrow a \leq c \rightarrow b$ and $b \rightarrow c \leq a \rightarrow c$.

(2) $(a \rightarrow b) \otimes(b \rightarrow a) \leq a \sim b \leq(a \rightarrow b) \wedge(b \rightarrow a)$.

(3) $a \otimes b \leq a \wedge b \leq a, b$ and $b \otimes a \leq a \wedge b \leq a, b$. 
(4) $a \rightarrow b=a \rightarrow(a \wedge b)$.

(5) $a \sim d \leq(a \sim c) \sim(d \sim c)$.

(6) $a \sim b=b \sim a$.

(7) $a \sim d \leq(a \wedge b) \sim(d \wedge b)$.

(8) $(a \rightarrow b) \otimes(b \rightarrow c) \leq a \rightarrow c$.

(9) $a \sim b \leq a \rightarrow b$ and $a \rightarrow a=1$.

(10) $a \rightarrow b \leq(a \wedge c) \rightarrow(b \wedge c)$.

(11) $a \rightarrow b \leq(b \rightarrow c) \rightarrow(a \rightarrow c)$.

Definition 2.3 A subset $F$ of an EQ-algebra $\mathcal{L}$ is called a prefilter of $\mathcal{L}$ if it satisfies the following conditions:

$$
\begin{aligned}
& 1 \in F, \\
& (\forall a, b \in L)(a \rightarrow b \in F, a \in F \Rightarrow b \in F) .
\end{aligned}
$$

Definition 2.4 A subset $F$ of an EQ-algebra $\mathcal{L}$ is called a filter of $\mathcal{L}$ if it is a prefilter of $\mathcal{L}$ with the following additional condition:

$(\forall a, b, c \in L)(a \rightarrow b \in F \Rightarrow(a \otimes c) \rightarrow(b \otimes c) \in F,(c \otimes a) \rightarrow(c \otimes b) \in F)$

Definition 2.5 A prefilter (resp. filter) $F$ of an EQ-algebra $\mathcal{L}$ is said to be positive implicative if the following assertion is valid:

$$
(\forall x, y, z \in L)(x \rightarrow(y \rightarrow z) \in F, x \rightarrow y \in F \Rightarrow x \rightarrow z \in F) .
$$

Torra [15] defined hesitant fuzzy sets in terms of a function that returns a set of membership values for each element in the domain. This is formally defined in the next definition.

Definition 2.6 ([15] ) Let $X$ be a reference set. Then we define hesitant fuzzy set on $X$ in terms of a function $\mathcal{H}$ that when applied to $X$ returns a subset of $[0,1]$.

For a hesitant fuzzy set $\mathcal{H}$ on $L$ and $x, y, z \in L$, we use the notations $\mathcal{H}_{x}:=\mathcal{H}(x), \mathcal{H}_{x}^{y}:=\mathcal{H}(x) \cap \mathcal{H}(y)$ and $\mathcal{H}_{x}^{y}[z]:=\mathcal{H}(x) \cap \mathcal{H}(y) \cap \mathcal{H}(z)$. It is clear that $\mathcal{H}_{x}^{y}=\mathcal{H}_{y}^{x}$, and

$$
\mathcal{H}_{x}=\mathcal{H}_{y} \Leftrightarrow \mathcal{H}_{x} \subseteq \mathcal{H}_{y}, \mathcal{H}_{y} \subseteq \mathcal{H}_{x}
$$

for all $x, y \in L$. 


\section{$3 \quad$ Hesitant fuzzy prefilters (filters)}

In what follows, we take a commutative EQ-algebra $\mathcal{L}$ as a reference set.

Definition 3.1 A hesitant fuzzy set $\mathcal{H}$ on $L$ is called an hesitant fuzzy prefilter of $\mathcal{L}$ if the following assertions are valid.

$$
\begin{aligned}
& (\forall x \in L)\left(\mathcal{H}_{x} \subseteq \mathcal{H}_{1}\right), \\
& (\forall x, y \in L)\left(\mathcal{H}_{x \rightarrow y}^{x} \subseteq \mathcal{H}_{y}\right) .
\end{aligned}
$$

Example 3.2 Let $E=\{0, a, b, 1\}$ be a chain. We define two binary operations ' $\otimes$ ' and ' $\sim$ ' by the Table 1 and Table 2 , respectively.

Table 1: Cayley table for the binary operations ' $\otimes$ '

\begin{tabular}{l|llll}
\hline$\otimes$ & 0 & $a$ & $b$ & 1 \\
\hline 0 & 0 & 0 & 0 & 0 \\
$a$ & 0 & 0 & 0 & $a$ \\
$b$ & 0 & 0 & 0 & $b$ \\
1 & 0 & $a$ & $b$ & 1 \\
\hline
\end{tabular}

Table 2: Cayley table for the binary operations ' $\sim$ '

\begin{tabular}{c|cccc}
\hline$\sim$ & 0 & $a$ & $b$ & 1 \\
\hline 0 & 1 & $a$ & $a$ & $a$ \\
$a$ & $a$ & 1 & $b$ & $b$ \\
$b$ & $a$ & $b$ & 1 & 1 \\
1 & $a$ & $b$ & 1 & 1 \\
\hline
\end{tabular}

Then $\mathcal{L}:=(L, \wedge, \otimes, \sim, 1)$ is an EQ-algebra (see [6]). The derived operation " $\rightarrow$ " is described by the Table 3 .

Define a hesitant fuzzy set $\mathcal{H}$ on $L$ as follows:

$$
\mathcal{H}: L \rightarrow \mathcal{P}([0,1]), x \mapsto \begin{cases}\{0.2,0.5,0.6,0.8\} & \text { if } x \in\{0, a\} \\ {[0.2,0.5] \cup[0.6,0.8]} & \text { if } x=b, \\ {[0.2,0.8]} & \text { if } x=1 .\end{cases}
$$

It is routine to check that $\mathcal{H}$ is a hesitant fuzzy prefilter of $\mathcal{L}$. 
Table 3: Cayley table for the implication ' $\rightarrow$ '

\begin{tabular}{c|cccc}
\hline$\rightarrow$ & 0 & $a$ & $b$ & 1 \\
\hline 0 & 1 & 1 & 1 & 1 \\
$a$ & $a$ & 1 & 1 & 1 \\
$b$ & $a$ & $b$ & 1 & 1 \\
1 & $a$ & $b$ & 1 & 1 \\
\hline
\end{tabular}

Definition 3.3 A hesitant fuzzy set $\mathcal{H}$ on $L$ is called an hesitant fuzzy filter of $\mathcal{L}$ if it is a hesitant fuzzy prefilter of $\mathcal{L}$ that satisfies the additional condition:

$$
(\forall x, y, z \in L)\left(\mathcal{H}_{x \rightarrow y} \subseteq \mathcal{H}_{(x \otimes z) \rightarrow(y \otimes z)}\right) .
$$

Example 3.4 Let $E=\{0, a, b, 1\}$ be a chain. We define two binary operations ' $\otimes$ ' and ' $\sim$ ' by the Table 4 and Table 5 , respectively.

Table 4: Cayley table for the binary operations ' $\otimes$ '

\begin{tabular}{l|llll}
\hline$\otimes$ & 0 & $a$ & $b$ & 1 \\
\hline 0 & 0 & 0 & 0 & 0 \\
$a$ & 0 & $a$ & $a$ & $a$ \\
$b$ & 0 & $a$ & $b$ & $b$ \\
1 & 0 & $a$ & $b$ & 1 \\
\hline
\end{tabular}

Table 5: Cayley table for the binary operations ' $\sim$

\begin{tabular}{c|cccc}
\hline$\sim$ & 0 & $a$ & $b$ & 1 \\
\hline 0 & 1 & 0 & 0 & 0 \\
$a$ & 0 & 1 & $a$ & $a$ \\
$b$ & 0 & $a$ & 1 & 1 \\
1 & 0 & $a$ & 1 & 1 \\
\hline
\end{tabular}

Then $\mathcal{L}:=(L, \wedge, \otimes, \sim, 1)$ is an EQ-algebra (see [6]). The derived operation " $\rightarrow$ " is described by the Table 6 . 
Table 6: Cayley table for the implication ' $\rightarrow$ '

\begin{tabular}{r|llll}
\hline$\rightarrow$ & 0 & $a$ & $b$ & 1 \\
\hline 0 & 1 & 1 & 1 & 1 \\
$a$ & 0 & 1 & 1 & 1 \\
$b$ & 0 & $a$ & 1 & 1 \\
1 & 0 & $a$ & 1 & 1 \\
\hline
\end{tabular}

Define a hesitant fuzzy set $\mathcal{H}$ on $L$ as follows:

$$
\mathcal{H}: L \rightarrow \mathcal{P}([0,1]), x \mapsto \begin{cases}{[0.4,0.6]} & \text { if } x \in\{0, a\} \\ (0.3,0.7] & \text { if } x \in\{b, 1\}\end{cases}
$$

It is routine to check that $\mathcal{H}$ is a hesitant fuzzy filter of $\mathcal{L}$.

Theorem 3.5 A hesitant fuzzy set $\mathcal{H}$ on $L$ is a hesitant fuzzy prefilter of $\mathcal{L}$ if and only if the set

$$
\mathcal{L}(\mathcal{H} ; \gamma):=\left\{x \in E \mid \gamma \subseteq \mathcal{H}_{x}\right\}
$$

which is called the hesitant level set of $\mathcal{H}$, is a prefilter of $\mathcal{L}$ for all $\gamma \in \mathcal{P}(U)$ with $\mathcal{L}(\mathcal{H} ; \gamma) \neq \emptyset$.

Proof. Assume that the hesitant level set $\mathcal{L}(\mathcal{H} ; \gamma)$ of $\mathcal{H}$ is a hesitant fuzzy prefilter of $\mathcal{L}$ for all $\gamma \in \mathcal{P}(U)$ with $\mathcal{L}(\mathcal{H} ; \gamma) \neq \emptyset$. For any $x \in L$, let $\mathcal{H}_{x}=\varepsilon_{x}$. Then $x \in \mathcal{L}\left(\mathcal{H} ; \varepsilon_{x}\right)$. Since $\mathcal{L}\left(\mathcal{H} ; \varepsilon_{x}\right)$ is a hesitant fuzzy prefilter of $\mathcal{L}$, we have $1 \in \mathcal{L}\left(\mathcal{H} ; \varepsilon_{x}\right)$ and so $\mathcal{H}_{x}=\varepsilon_{x} \subseteq \mathcal{H}_{1}$ for all $x \in L$. For any $x, y \in L$, let $\mathcal{H}_{x \rightarrow y}^{x}=$ $\delta$. Then $\mathcal{H}_{x} \supseteq \delta$ and $\mathcal{H}_{x \rightarrow y} \supseteq \delta$, that is, $x \in \mathcal{L}(\mathcal{H} ; \delta)$ and $x \rightarrow y \in \mathcal{L}(\mathcal{H} ; \delta)$. It follows from $(3)$ that $y \in \mathcal{L}(\mathcal{H} ; \delta)$ and that $\mathcal{H}_{y} \supseteq \delta=\mathcal{H}_{x \rightarrow y}^{x}$.

Conversely, let $\mathcal{H}$ be a hesitant fuzzy prefilter of $\mathcal{L}$. Let $x, y \in L$ and $\varepsilon \in \mathcal{P}(U)$ be such that $x \in \mathcal{L}(\mathcal{H} ; \varepsilon)$ and $x \rightarrow y \in \mathcal{L}(\mathcal{H} ; \varepsilon)$. Then $\varepsilon \subseteq \mathcal{H}_{x}$ and $\varepsilon \subseteq \mathcal{H}_{x \rightarrow y}$. It follows from (6) and (7) that $\varepsilon \subseteq \mathcal{H}_{x \rightarrow y}^{x} \subseteq \mathcal{H}_{y} \subseteq \mathcal{H}_{1}$. Hence $1 \in \mathcal{L}(\mathcal{H} ; \varepsilon)$ and $y \in \mathcal{L}(\mathcal{H} ; \varepsilon)$. Therefore $\mathcal{L}(\mathcal{H} ; \varepsilon)$ is a prefilter of $\mathcal{L}$ for all $\varepsilon \in \mathcal{P}(U)$ with $\mathcal{L}(\mathcal{H} ; \varepsilon) \neq \emptyset$.

Theorem 3.6 A hesitant fuzzy set $\mathcal{H}$ on $L$ is a hesitant fuzzy filter of $\mathcal{L}$ if and only if the hesitant level set $\mathcal{L}(\mathcal{H} ; \gamma)$ of $\mathcal{H}$ is a filter of $\mathcal{L}$ for all $\gamma \in \mathcal{P}(U)$ with $\mathcal{L}(\mathcal{H} ; \gamma) \neq \emptyset$.

Proof. Let $\mathcal{H}$ be a hesitant fuzzy set on $L$ such that its hesitant level set $\mathcal{L}(\mathcal{H} ; \gamma)$ is a filter of $\mathcal{L}$ for all $\gamma \in \mathcal{P}(U)$ with $\mathcal{L}(\mathcal{H} ; \gamma) \neq \emptyset$. Since $\mathcal{L}(\mathcal{H} ; \gamma)$ is a 
prefilter of $\mathcal{L}$, we know that $\mathcal{H}$ is a hesitant fuzzy prefilter of $\mathcal{L}$ by Theorem 3.5. Let $x, y \in L$ and $\tau \in \mathcal{P}(U)$ be such that $\mathcal{H}_{x \rightarrow y}=\tau$. Then $x \rightarrow y \in \mathcal{L}(\mathcal{H} ; \tau)$. Since $\mathcal{L}(\mathcal{H} ; \tau)$ is a filter of $\mathcal{L}$, we have $(x \otimes z) \rightarrow(y \otimes z) \in \mathcal{L}(\mathcal{H} ; \tau)$ for all $z \in L$. It follows that

$$
\mathcal{H}_{x \rightarrow y}=\tau \subseteq \mathcal{H}_{(x \otimes z) \rightarrow(y \otimes z)}
$$

for all $x, y, z \in L$. Therefore $\mathcal{H}$ is a hesitant fuzzy filter of $\mathcal{L}$.

Conversely, suppose that $\mathcal{H}$ is a hesitant fuzzy filter of $\mathcal{L}$. Then $\mathcal{H}$ is a hesitant fuzzy prefilter of $\mathcal{L}$, and so $\mathcal{L}(\mathcal{H} ; \gamma)$ is a prefilter of $\mathcal{L}$ for all $\gamma \in \mathcal{P}(U)$ with $\mathcal{L}(\mathcal{H} ; \gamma) \neq \emptyset$ by Theorem 3.5. Let $x, y \in L$ be such that $x \rightarrow y \in \mathcal{L}(\mathcal{H} ; \gamma)$. Then

$$
\gamma \subseteq \mathcal{H}_{x \rightarrow y} \subseteq \mathcal{H}_{(x \otimes z) \rightarrow(y \otimes z)}
$$

by $(8)$, and so $(x \otimes z) \rightarrow(y \otimes z) \in \mathcal{L}(\mathcal{H} ; \gamma)$. Hence $\mathcal{L}(\mathcal{H} ; \gamma)$ is a filter of $\mathcal{L}$ for all $\gamma \in \mathcal{P}(U)$ with $\mathcal{L}(\mathcal{H} ; \gamma) \neq \emptyset$.

Proposition 3.7 For every hesitant fuzzy prefilter $\mathcal{H}$ of $\mathcal{L}$, we have the following properties:

(1) If $x \leq y$, then $\mathcal{H}_{x} \subseteq \mathcal{H}_{y}$,

(2) $\mathcal{H}_{x \sim y}^{x} \subseteq \mathcal{H}_{y}$

(3) $\mathcal{H}_{x}^{y} \subseteq \mathcal{H}_{x \wedge y}$

(4) $\mathcal{H}_{x \sim y}^{y \sim z} \subseteq \mathcal{H}_{x \sim z}$

(5) $\mathcal{H}_{1 \sim x}=\mathcal{H}_{x}$

(6) $\mathcal{H}_{(x \rightarrow y) \otimes(y \rightarrow x)} \subseteq \mathcal{H}_{x \sim y} \subseteq \mathcal{H}_{(x \rightarrow y) \wedge(y \rightarrow x)}$,

(7) $\mathcal{H}_{y} \subseteq \mathcal{H}_{x \rightarrow y}$

(8) $\mathcal{H}_{(x \rightarrow(a \wedge b)) \otimes(a \sim y)} \subseteq \mathcal{H}_{x \rightarrow(y \wedge b)}$,

(9) $\mathcal{H}_{(x \rightarrow y) \otimes(y \sim z)} \subseteq \mathcal{H}_{x \rightarrow z}$,

for all $a, b, x, y, z \in L$.

Proof. (1) Let $x, y \in L$ be such that $x \leq y$. Then $x \rightarrow y=1$ by Proposition 2.2(1). It follows from (6) and (7) that $\mathcal{H}_{x}=\mathcal{H}_{1}^{x}=\mathcal{H}_{x \rightarrow y}^{x} \subseteq \mathcal{H}_{y}$.

(2) Let $x, y \in L$. Note that $x \sim y \leq x \rightarrow y$ by (2) and (3) of Proposition 2.2. Using (7) and item (1) implies that $\mathcal{H}_{x \sim y}^{x} \subseteq \mathcal{H}_{x \rightarrow y}^{x} \subseteq \mathcal{H}_{y}$.

(3) Note that $y \leq x \rightarrow y=x \rightarrow(x \wedge y)$ for all $x, y \in L$. It follows from (7) and item (1) that

$$
\mathcal{H}_{x}^{y} \subseteq \mathcal{H}_{x \rightarrow(x \wedge y)}^{x} \subseteq \mathcal{H}_{x \wedge y}
$$

for all $x, y \in L$. 
(4) Since $x \sim y=y \sim x \leq(y \sim z) \sim(x \sim z)$ by (6) and (5) of Proposition 2.2 , it follows from items (1) and (2) that

$$
\mathcal{H}_{y \sim z}^{x \sim y} \subseteq \mathcal{H}_{(y \sim z) \sim(x \sim z)}^{y \sim z} \subseteq \mathcal{H}_{x \sim z}
$$

for all $x, y, z \in L$.

(5) Using item (2) and (6), we have $\mathcal{H}_{x} \supseteq \mathcal{H}_{1 \sim x}^{1}=\mathcal{H}_{1 \sim x}$ for all $x \in L$. Since $x \leq x \sim 1$ for all $x \in L$, it follows from (1) and Proposition 2.2(6) that $\mathcal{H}_{x} \subseteq \mathcal{H}_{x \sim 1}=\mathcal{H}_{1 \sim x}$ for all $x \in L$. Therefore (5) holds.

(6) follows from Proposition 2.2(2) and item (1).

(7) For any $x, y \in L$, we have

$$
\mathcal{H}_{y} \subseteq \mathcal{H}_{1 \rightarrow y}=\mathcal{H}_{(1 \wedge y) \sim 1} \subseteq \mathcal{H}_{(x \wedge y) \sim x}=\mathcal{H}_{x \rightarrow y}
$$

(8) Using the commutativity and associativity of $\wedge$, (L4), the definition of implication $(\rightarrow)$ and item $(1)$, we have

$$
\begin{aligned}
\mathcal{H}_{(x \rightarrow(a \wedge b)) \otimes(a \sim y)} & =\mathcal{H}_{((x \wedge(a \wedge b)) \sim x) \otimes(a \sim y)} \\
& =\mathcal{H}_{((a \wedge(b \wedge x)) \sim x) \otimes(y \sim a)} \\
& \subseteq \mathcal{H}_{x \sim(y \wedge(b \wedge x))} \\
& =\mathcal{H}_{(x \wedge(y \wedge b)) \sim x} \\
& =\mathcal{H}_{x \rightarrow(y \wedge b)}
\end{aligned}
$$

for all $x, y, a, b \in L$.

(9) Using item (8), (L6) and (1), we get

$$
\begin{aligned}
\mathcal{H}_{(x \rightarrow y) \otimes(y \sim z)} & =\mathcal{H}_{(x \rightarrow(y \wedge y)) \otimes(y \sim z)} \\
& \subseteq \mathcal{H}_{x \rightarrow(z \wedge y)} \\
& =\mathcal{H}_{(x \wedge(z \wedge y)) \sim x} \\
& \subseteq \mathcal{H}_{(x \wedge z) \sim x} \\
& =\mathcal{H}_{(z \wedge x) \sim x} \\
& =\mathcal{H}_{x \rightarrow z}
\end{aligned}
$$

for all $x, y, z \in L$.

Proposition 3.8 Every hesitant fuzzy prefilter $\mathcal{H}$ of $\mathcal{L}$ satisfies the following assertions.

(1) $\mathcal{H}_{x \sim y}^{a \sim b} \subseteq \mathcal{H}_{(a \wedge x) \sim(b \wedge y)}^{(a \sim x) \sim(b \sim y)}[(a \rightarrow x) \sim(b \rightarrow y)]$,

(2) $\mathcal{H}_{x \otimes y} \subseteq \mathcal{H}_{x}^{y}$

(3) If $x \leq y$, then $\mathcal{H}_{\tilde{x}} \subseteq \mathcal{H}_{\tilde{y}}$ and $\mathcal{H}_{a \rightarrow x} \subseteq \mathcal{H}_{a \rightarrow y}$, 
for all $a, b, x, y \in L$.

Proof. (1) Let $a, b, x, y \in L$. By Proposition 2.2(7), we have

$$
a \sim b \leq(a \wedge x) \sim(b \wedge x) \text { and } x \sim y \leq(b \wedge x) \sim(b \wedge y) .
$$

It follows from Proposition 3.7(1) that

$$
\mathcal{H}_{a \sim b} \subseteq \mathcal{H}_{(a \wedge x) \sim(b \wedge x)} \text { and } \mathcal{H}_{x \sim y} \subseteq \mathcal{H}_{(b \wedge x) \sim(b \wedge y)} .
$$

and from Proposition 3.7(4) that

$$
\mathcal{H}_{x \sim y}^{a \sim b} \subseteq \mathcal{H}_{(b \wedge x) \sim(b \wedge y)}^{(a \wedge x) \sim(b \wedge x)} \subseteq \mathcal{H}_{(a \wedge x) \sim(b \wedge y)} .
$$

Using items (5) and (6) of Proposition 2.2, we get

$$
a \sim b \leq(a \sim x) \sim(b \sim x) \text { and } x \sim y \leq(b \sim x) \sim(b \sim y)
$$

for all $a, b, x, y \in L$. Hence we have

$$
\mathcal{H}_{x \sim y}^{a \sim b} \subseteq \mathcal{H}_{(a \sim x) \sim(b \sim y)}
$$

by the similar way to the proof of (9).

Using (1), (9) and (10), we get $\mathcal{H}_{x \sim y}^{a \sim b} \subseteq \mathcal{H}_{(a \rightarrow x) \sim(b \rightarrow y)}$. Therefore

$$
\mathcal{H}_{x \sim y}^{a \sim b} \subseteq \mathcal{H}_{(a \wedge x) \sim(b \wedge y)}^{(a \sim x)(b \sim y)}[(a \rightarrow x) \sim(b \rightarrow y)] .
$$

(2) Using Proposition 2.2(3) and Proposition 3.7(1), we have $\mathcal{H}_{x \otimes y} \subseteq \mathcal{H}_{x}^{y}$ for all $x, y \in L$.

(3) Let $x, y \in L$ be such that $x \leq y$. Then $x \wedge y=x$, and so

$$
\begin{aligned}
\mathcal{H}_{\tilde{x}} & =\mathcal{H}_{x \sim 1}=\mathcal{H}_{(1 \wedge x) \sim 1} \\
& =\mathcal{H}_{(1 \wedge(x \wedge y)) \sim 1}=\mathcal{H}_{(1 \wedge y \wedge x) \sim 1} \\
& \subseteq \mathcal{H}_{(1 \wedge y) \sim 1}=\mathcal{H}_{y \sim 1}=\mathcal{H}_{\tilde{y}}
\end{aligned}
$$

and

$$
\begin{aligned}
\mathcal{H}_{a \rightarrow x} & =\mathcal{H}_{(a \wedge x) \sim a}=\mathcal{H}_{(a \wedge(x \wedge y)) \sim a} \\
& =\mathcal{H}_{(a \wedge y \wedge x) \sim a} \subseteq \mathcal{H}_{(a \wedge y) \sim a}=\mathcal{H}_{a \rightarrow y}
\end{aligned}
$$

by (L1), (L6) and Proposition 3.7(1).

Theorem 3.9 For a hesitant fuzzy set $\mathcal{H}$ on $L$, the following are equivalent.

(1) $\mathcal{H}$ is a hesitant fuzzy prefilter of $\mathcal{L}$. 
(2) $(\forall x, y, z \in L)\left(x \leq y \rightarrow z \Rightarrow \mathcal{H}_{x}^{y} \subseteq \mathcal{H}_{z}\right)$.

(3) $(\forall x, y, z \in L)\left(x \rightarrow(y \rightarrow z)=1 \Rightarrow \mathcal{H}_{x}^{y} \subseteq \mathcal{H}_{z}\right)$.

Proof. $(1) \Rightarrow(2)$ Let $x, y, z \in L$ be such that $x \leq y \rightarrow z$. Then $\mathcal{H}_{x} \subseteq \mathcal{H}_{y \rightarrow z}$ by Proposition 3.7(1). Using (7), we get

$$
\mathcal{H}_{x}^{y} \subseteq \mathcal{H}_{y \rightarrow z}^{y} \subseteq \mathcal{H}_{z} .
$$

$(2) \Rightarrow(3)$ Let $x, y, z \in L$ be such that $x \rightarrow(y \rightarrow z)=1$. Then

$$
x \leq 1=x \rightarrow(y \rightarrow z),
$$

and so $\mathcal{H}_{x} \subseteq \mathcal{H}_{y \rightarrow z}$ by item (2). Since $y \rightarrow z \leq y \rightarrow z$, it follows from item (2) that

$$
\mathcal{H}_{x}^{y} \subseteq \mathcal{H}_{y \rightarrow z}^{y} \subseteq \mathcal{H}_{z} .
$$

$(3) \Rightarrow(1)$ Since $x \rightarrow(x \rightarrow 1)=1$ for all $x \in L$, it follows from item (3) that $\mathcal{H}_{x} \subseteq \mathcal{H}_{1}$ for all $x \in L$. Note that $(x \rightarrow y) \rightarrow(x \rightarrow y)=1$ for all $x, y \in L$. Thus

$$
\mathcal{H}_{x \rightarrow y}^{x} \subseteq \mathcal{H}_{y}
$$

for all $x, y \in L$ by item (3). Therefore $\mathcal{H}$ is a hesitant fuzzy prefilter of $\mathcal{L}$.

Proposition 3.10 For any hesitant fuzzy filter $\mathcal{H}$ of $\mathcal{L}$, the following assertions are valid.

(1) $\mathcal{H}_{x \otimes y}=\mathcal{H}_{x}^{y}$

(2) $\mathcal{H}_{y \rightarrow z}^{x \rightarrow y} \subseteq \mathcal{H}_{x \rightarrow z}$,

(3) $\mathcal{H}_{x \sim y} \subseteq \mathcal{H}_{(x \otimes z) \sim(y \otimes z)}$,

for all $x, y, z \in L$.

Proof. (1) The inclusion $\mathcal{H}_{x \otimes y} \subseteq \mathcal{H}_{x}^{y}$ follows from Proposition 3.8(2). Note that $y \leq 1 \rightarrow y$ for all $y \in L$. It follows from Proposition 3.7(1) and (8) that

$$
\mathcal{H}_{y} \subseteq \mathcal{H}_{1 \rightarrow y} \subseteq \mathcal{H}_{(x \otimes 1) \rightarrow(x \otimes y)}=\mathcal{H}_{x \rightarrow(x \otimes y)}
$$

and from (7) that $\mathcal{H}_{x}^{y} \subseteq \mathcal{H}_{x \rightarrow(x \otimes y)}^{x} \subseteq \mathcal{H}_{x \otimes y}$ for all $x, y \in L$.

(2) Combining Proposition 2.2(8), Proposition 3.7(1) and item (1) induces

$$
\mathcal{H}_{y \rightarrow z}^{x \rightarrow y}=\mathcal{H}_{(x \rightarrow y) \otimes(y \rightarrow z)} \subseteq \mathcal{H}_{x \rightarrow z}
$$

for all $x, y, z \in L$.

(3) Using items (6) and (9) of Proposition 2.2, (8), item (1) and Proposition $3.7(1)$, we have

$$
\begin{aligned}
\mathcal{H}_{x \sim y} & \subseteq \mathcal{H}_{y \rightarrow x}^{x \rightarrow y} \subseteq \mathcal{H}_{(y \otimes z) \rightarrow(x \otimes z)}^{(x \otimes z) \rightarrow(y \otimes z)} \\
& =\mathcal{H}_{((x \otimes z) \rightarrow(y \otimes z)) \otimes((y \otimes z) \rightarrow(x \otimes z))} \\
& \subseteq \mathcal{H}_{(x \otimes z) \sim(y \otimes z)}
\end{aligned}
$$

for all $x, y, z \in L$. 


\section{Positive implicative hesitant fuzzy prefilters (filters)}

Definition 4.1 A hesitant fuzzy set $\mathcal{H}$ on $L$ is called a positive implicative hesitant fuzzy prefilter (filter) of $\mathcal{L}$ if it is a hesitant fuzzy prefilter (filter) of $\mathcal{L}$ that satisfies an additional condition:

$$
(\forall x, y, z \in L)\left(\mathcal{H}_{x \rightarrow(y \rightarrow z)}^{x \rightarrow y} \subseteq \mathcal{H}_{x \rightarrow z}\right) .
$$

Example 4.2 The hesitant fuzzy filter $\mathcal{H}$ in Example 3.4 is positive implicative, but the hesitant fuzzy prefilter $\mathcal{H}$ in Example 3.2 is not positive implicative since

$$
\mathcal{H}_{b \rightarrow(1 \rightarrow 0)}^{b \rightarrow 1}=\mathcal{H}_{b}^{b}=\mathcal{H}_{b} \nsubseteq \mathcal{H}_{a}=\mathcal{H}_{b \rightarrow 0}
$$

Theorem 4.3 A hesitant fuzzy set $\mathcal{H}$ on $L$ is a positive implicative hesitant fuzzy prefilter (filter) of $\mathcal{L}$ if and only if the hesitant level set $\mathcal{L}(\mathcal{H} ; \gamma)$ of $\mathcal{H}$ is a positive implicative prefilter (filter) of $\mathcal{L}$ for all $\gamma \in \mathcal{P}(U)$ with $\mathcal{L}(\mathcal{H} ; \gamma) \neq \emptyset$.

Proof. Assume that $\mathcal{H}$ is a positive implicative hesitant fuzzy prefilter (filter) of $\mathcal{L}$. Then $\mathcal{L}(\mathcal{H} ; \varepsilon)$ is a prefilter (filter) of $\mathcal{L}$ for all $\varepsilon \in \mathcal{P}(U)$ with $\mathcal{L}(\mathcal{H} ; \varepsilon) \neq \emptyset$. Let $x, y, z \in L$ be such that

$$
x \rightarrow(y \rightarrow z) \in \mathcal{L}(\mathcal{H} ; \varepsilon) \text { and } x \rightarrow y \in \mathcal{L}(\mathcal{H} ; \varepsilon) .
$$

Then $\varepsilon \subseteq \mathcal{H}_{x \rightarrow(y \rightarrow z)}$ and $\varepsilon \subseteq \mathcal{H}_{x \rightarrow y}$. It follows from (11) that

$$
\varepsilon \subseteq \mathcal{H}_{x \rightarrow(y \rightarrow z)}^{x \rightarrow y} \subseteq \mathcal{H}_{x \rightarrow z}
$$

and that $x \rightarrow z \in \mathcal{L}(\mathcal{H} ; \varepsilon)$. Hence $\mathcal{L}(\mathcal{H} ; \varepsilon)$ is a positive implicative prefilter (filter) of $\mathcal{L}$ for all $\varepsilon \in \mathcal{P}(U)$ with $\mathcal{L}(\mathcal{H} ; \varepsilon) \neq \emptyset$.

Suppose that the hesitant level set $\mathcal{L}(\mathcal{H} ; \gamma)$ of $\mathcal{H}$ is a positive implicative prefilter (filter) of $\mathcal{L}$ for all $\gamma \in \mathcal{P}(U)$ with $\mathcal{L}(\mathcal{H} ; \gamma) \neq \emptyset$. Then $\mathcal{H}$ is a hesitant fuzzy prefilter (filter) of $\mathcal{L}$. Let $x, y, z \in L$ be such that $\mathcal{H}_{x \rightarrow y \rightarrow z)}^{x \rightarrow y}=\varepsilon$. Then $x \rightarrow(y \rightarrow z) \in \mathcal{L}(\mathcal{H} ; \varepsilon)$ and $x \rightarrow y \in \mathcal{L}(\mathcal{H} ; \varepsilon)$, which implies from (5) that $x \rightarrow z \in \mathcal{L}(\mathcal{H} ; \varepsilon)$. Thus

$$
\mathcal{H}_{x \rightarrow(y \rightarrow z)}^{x \rightarrow y}=\varepsilon \subseteq \mathcal{H}_{x \rightarrow z}
$$

and therefore $\mathcal{H}$ is a positive implicative hesitant fuzzy prefilter (filter) of $\mathcal{L}$.

Proposition 4.4 Every positive implicative hesitant fuzzy prefilter $\mathcal{H}$ of $\mathcal{L}$ satisfies the following assertion:

$$
(\forall x, y \in L)\left(\mathcal{H}_{((x \rightarrow y) \wedge x) \rightarrow y}=\mathcal{H}_{1}\right) .
$$


Proof. If $\mathcal{H}$ is a positive implicative hesitant fuzzy prefilter of $\mathcal{L}$, then it is a hesitant fuzzy prefilter of $\mathcal{L}$. Since $(x \rightarrow y) \wedge x \leq x$ and $(x \rightarrow y) \wedge x \leq x \rightarrow y$ for all $x, y \in L$, we have $((x \rightarrow y) \wedge x) \rightarrow x=1$ and $((x \rightarrow y) \wedge x) \rightarrow(x \rightarrow y)=1$. It follows from (11) that

$$
\mathcal{H}_{1}=\mathcal{H}_{((x \rightarrow y) \wedge x) \rightarrow(x \rightarrow y)}^{((x \rightarrow y) \wedge x) \rightarrow x} \subseteq \mathcal{H}_{((x \rightarrow y) \wedge x) \rightarrow y}
$$

and that $\mathcal{H}_{((x \rightarrow y) \wedge x) \rightarrow y}=\mathcal{H}_{1}$ for all $x, y \in L$.

Corollary 4.5 If $\mathcal{H}$ is a positive implicative hesitant fuzzy prefilter $\mathcal{H}$ of $\mathcal{L}$, then

$$
\mathcal{H}_{(1 \rightarrow x) \rightarrow x}=\mathcal{H}_{1}
$$

for all $x \in L$.

Corollary 4.6 If $\mathcal{H}$ is a positive implicative hesitant fuzzy prefilter $\mathcal{H}$ of $\mathcal{L}$, then

$$
\mathcal{H}_{(x \otimes(x \rightarrow y)) \rightarrow y}=\mathcal{H}_{1}
$$

for all $x, y \in L$.

Proof. Using (1) and (3) in Proposition 2.2, we have

$$
((x \rightarrow y) \wedge x) \rightarrow y \leq(x \otimes(x \rightarrow y)) \rightarrow y
$$

for all $x, y \in L$. It follows from (12) and Proposition 3.7(1) that

$$
\mathcal{H}_{1}=\mathcal{H}_{((x \rightarrow y) \wedge x) \rightarrow y} \subseteq \mathcal{H}_{(x \otimes(x \rightarrow y)) \rightarrow y}
$$

and from (6) that $\mathcal{H}_{(x \otimes(x \rightarrow y)) \rightarrow y}=\mathcal{H}_{1}$ for all $x, y \in L$.

We provide a condition for a hesitant fuzzy filter to be a positive implicative hesitant fuzzy filter.

Theorem 4.7 If a hesitant fuzzy filter of $\mathcal{L}$ satisfies the condition (12), then it is a positive implicative hesitant fuzzy filter of $\mathcal{L}$.

Proof. Let $\mathcal{H}$ be a hesitant fuzzy filter of $\mathcal{L}$ that satisfies the condition (12). Using (4) and (10) in Proposition 2.2, we have

$$
x \rightarrow(y \rightarrow z) \leq(x \wedge y) \rightarrow((y \rightarrow z) \wedge y) \text { and } x \rightarrow y=x \rightarrow(x \wedge y)
$$

for all $x, y, z \in L$. It follows from (6), Proposition 3.7(1), Proposition 3.10(2) and (12) that

$$
\begin{aligned}
& \mathcal{H}_{x \rightarrow(y \rightarrow z)}^{x \rightarrow y}=\mathcal{H}_{x \rightarrow(y \rightarrow z)}^{x \rightarrow y}[1] \subseteq \mathcal{H}_{(x \wedge y) \rightarrow((y \rightarrow z) \wedge y)}^{x \rightarrow(x \wedge y)}[1] \\
& \subseteq \mathcal{H}_{((y \rightarrow((y \rightarrow z) \wedge y) \wedge y) \rightarrow z}^{(x \rightarrow(x)} \subseteq \mathcal{H}_{x \rightarrow z}
\end{aligned}
$$

for all $x, y, z \in L$. Therefore $\mathcal{H}$ is a positive implicative hesitant fuzzy filter of $\mathcal{L}$. 
Corollary 4.8 A hesitant fuzzy set $\mathcal{H}$ on $L$ is a positive implicative hesitant fuzzy filter of $\mathcal{L}$ if and only if it is a hesitant fuzzy filter of $\mathcal{L}$ which satisfies the condition (12).

Theorem 4.9 Let $\mathcal{H}$ be a hesitant fuzzy prefilter (filter) of $\mathcal{L}$. Then $\mathcal{H}$ is positive implicative if and only if it satisfies:

$$
(\forall x, y \in L)\left(\mathcal{H}_{x \rightarrow(x \rightarrow y)} \subseteq \mathcal{H}_{x \rightarrow y}\right) .
$$

Proof. Assume that $\mathcal{H}$ is a positive implicative hesitant fuzzy prefilter of $\mathcal{L}$. If we take $y=x$ and $z=y$ in (11), then

$$
\mathcal{H}_{x \rightarrow(x \rightarrow y)}=\mathcal{H}_{x \rightarrow(x \rightarrow y)}^{1}=\mathcal{H}_{x \rightarrow(x \rightarrow y)}^{x \rightarrow x} \subseteq \mathcal{H}_{x \rightarrow y}
$$

for all $x, y \in L$.

Conversely, let $\mathcal{H}$ be a hesitant fuzzy prefilter of $\mathcal{L}$ satisfying (13). Using (13), (7), Proposition 2.2(11) and Proposition 3.7(1), we obtain

$$
\mathcal{H}_{x \rightarrow(y \rightarrow z)}^{x \rightarrow y} \subseteq \mathcal{H}_{((y \rightarrow z) \rightarrow(x \rightarrow z)) \rightarrow(x \rightarrow(x \rightarrow z))}^{(y \rightarrow z) \rightarrow(x \rightarrow z)} \subseteq \mathcal{H}_{x \rightarrow(x \rightarrow z)} \subseteq \mathcal{H}_{x \rightarrow z}
$$

for all $x, y, z \in L$. Therefore $\mathcal{H}$ is positive implicative.

Theorem 4.10 Let $\mathcal{H}$ be a hesitant fuzzy prefilter of $\mathcal{L}$ with an additional condition:

$$
(\forall x, y, z \in L)\left(\mathcal{H}_{x \rightarrow(y \rightarrow z)} \subseteq \mathcal{H}_{y \rightarrow(x \rightarrow z)}\right)
$$

Then $\mathcal{H}$ is positive implicative if and only if it satisfies:

$$
(\forall x, y, z \in L)\left(\mathcal{H}_{x \rightarrow(y \rightarrow z)} \subseteq \mathcal{H}_{(x \rightarrow y) \rightarrow(x \rightarrow z)}\right) .
$$

Proof. If $\mathcal{H}$ is positive implicative, then

$$
\begin{aligned}
& \mathcal{H}_{(x \rightarrow y) \rightarrow(x \rightarrow z)} \supseteq \mathcal{H}_{x \rightarrow((x \rightarrow y) \rightarrow z)} \\
& \supseteq \mathcal{H}_{x \rightarrow((y \rightarrow z)}^{x \rightarrow(y \rightarrow) \rightarrow((x \rightarrow y) \rightarrow z))} \\
& \supseteq \mathcal{H}_{x \rightarrow((x \rightarrow y) \rightarrow y)}^{x \rightarrow(y \rightarrow z)} \\
& \supseteq \mathcal{H}_{(x \rightarrow y) \rightarrow(x \rightarrow y)}^{x \rightarrow(y \rightarrow z)} \\
& =\mathcal{H}_{x \rightarrow(y \rightarrow z)}^{1}=\mathcal{H}_{x \rightarrow(y \rightarrow z)}
\end{aligned}
$$

for all $x, y, z \in L$ by (14), (11), Proposition 2.2(11), Proposition 2.2(2) and (6).

Conversely, assume that $\mathcal{H}$ satisfies the condition (15). Then

$$
\mathcal{H}_{x \rightarrow z} \supseteq \mathcal{H}_{(x \rightarrow y) \rightarrow(x \rightarrow z)}^{x \rightarrow y} \supseteq \mathcal{H}_{x \rightarrow(y \rightarrow z)}^{x \rightarrow y}
$$

for all $x, y, z \in L$ by (7) and (15). Therefore $\mathcal{H}$ is positive implicative. 
Theorem 4.11 Let $\mathcal{L}$ satisfy the following condition:

$$
(\forall x, y, z \in L)((x \otimes y) \rightarrow z \leq x \rightarrow(y \rightarrow z))
$$

Then a hesitant fuzzy set $\mathcal{H}$ on $L$ is a positive implicative hesitant fuzzy filter of $\mathcal{L}$ if and only if it is a hesitant fuzzy filter of $\mathcal{L}$ which satisfies the following condition:

$$
(\forall x, y \in L)\left(\mathcal{H}_{x \rightarrow(x \otimes x)}=\mathcal{H}_{1}=\mathcal{H}_{(x \otimes(x \rightarrow y)) \rightarrow y}\right)
$$

Proof. Assume that $\mathcal{H}$ is a positive implicative hesitant fuzzy filter of $\mathcal{L}$. Then it is a hesitant fuzzy filter of $\mathcal{L}$ and $\mathcal{H}((x \otimes(x \rightarrow y)) \rightarrow y)=\mathcal{H}_{1}$ for all $x, y \in L$ by Corollary 4.6. Using (16), Proposition 3.7(1), (6) and (11), we have

$$
\begin{aligned}
\mathcal{H}_{1} & =\mathcal{H}_{(x \otimes x) \rightarrow(x \otimes x)} \subseteq \mathcal{H}_{x \rightarrow(x \rightarrow(x \otimes x))} \\
& =\mathcal{H}_{x \rightarrow(x \rightarrow(x \otimes x))}^{1}=\mathcal{H}_{x \rightarrow(x \rightarrow(x \otimes x))}^{x \rightarrow x} \\
& \subseteq \mathcal{H}_{x \rightarrow(x \otimes x)},
\end{aligned}
$$

and so $\mathcal{H}_{x \rightarrow(x \otimes x)}=\mathcal{H}_{1}$ for all $x \in L$.

Conversely, let $\mathcal{H}$ be a hesitant fuzzy filter of $\mathcal{L}$ satisfying the condition (17). Using (8), we obtain

$$
\mathcal{H}_{x \rightarrow(y \rightarrow z)} \subseteq \mathcal{H}_{(x \otimes y) \rightarrow((y \rightarrow z) \otimes y)} \text { and } \mathcal{H}_{x \rightarrow y} \subseteq \mathcal{H}_{(x \otimes x) \rightarrow(x \otimes y)}
$$

for all $x, y, z \in L$. It follows from Proposition 3.10(2), (17), (6), that

$$
\begin{aligned}
\mathcal{H}_{x \rightarrow z} & \supseteq \mathcal{H}_{(x \otimes x) \rightarrow z}^{x \rightarrow(x \otimes x)}=\mathcal{H}_{(x \otimes x) \rightarrow z}^{1}=\mathcal{H}_{(x \otimes x) \rightarrow z} \\
& \supseteq \mathcal{H}_{(x \otimes x) \rightarrow(y \otimes(y \rightarrow z))}^{(y \otimes(y \rightarrow z) \rightarrow z} \\
& =\mathcal{H}_{(x \otimes x) \rightarrow(y \otimes(y \rightarrow z))}^{1} \\
& =\mathcal{H}_{(x \otimes x) \rightarrow(y \otimes(y \rightarrow z))} \\
& \supseteq \mathcal{H}_{(x \otimes y) \rightarrow((y \rightarrow z) \otimes y)}^{(x \otimes x) \rightarrow(x \otimes y)} \\
& \supseteq \mathcal{H}_{x \rightarrow(y \rightarrow z)}^{x \rightarrow y}
\end{aligned}
$$

for all $x, y, z \in L$. Hence $\mathcal{H}$ is a positive implicative hesitant fuzzy filter of $\mathcal{L}$.

Note that every residuated EQ-algebra satisfies the condition (16), but not vice versa (see [6]). Hence we have the following corollary.

Corollary 4.12 In a residuated EQ-algebra, a hesitant fuzzy set $\mathcal{H}$ on $L$ is a positive implicative hesitant fuzzy filter of $\mathcal{L}$ if and only if it is a hesitant fuzzy filter of $\mathcal{L}$ which satisfies the condition (17). 
Finally, we establish the extension property for a positive implicative hesitant fuzzy prefilter (filter).

Theorem 4.13 Let $\mathcal{H}$ and $\mathcal{G}$ be hesitant fuzzy filters of $\mathcal{L}$ such that $\mathcal{H}_{1}=\mathcal{G}_{1}$ and $\mathcal{H}_{x} \subseteq \mathcal{G}_{x}$ for all $x \in L$. If $\mathcal{H}$ is positive implicative, then so is $\mathcal{G}$.

Proof. For any $x, y \in L$, we have

$$
\mathcal{G}_{1}=\mathcal{H}_{1}=\mathcal{H}_{((x \rightarrow y) \wedge x) \rightarrow y} \subseteq \mathcal{G}_{((x \rightarrow y) \wedge x) \rightarrow y},
$$

and thus $\mathcal{G}_{((x \rightarrow y) \wedge x) \rightarrow y}=\mathcal{G}_{1}$ for all $x, y \in L$. Therefore $\mathcal{G}$ is a positive implicative hesitant fuzzy filter of $\mathcal{L}$ by Theorem 4.7 .

\section{References}

[1] J. D. Bashford and P. D. Jarvis, The genetic code as a periodic table: algebraic aspects, BioSystems, 57 (2000), 147-161. http://dx.doi.org/10.1016/s0303-2647(00)00097-6

[2] M. El-Zekey, Representable good EQ-algebras, Soft Computing 14(9) (2010) 1011-1023. http://dx.doi.org/10.1007/s00500-009-0491-4

[3] M. El-Zekey, V. Novák and R. Mesiar, On good EQalgebras, Fuzzy Sets and Systems 178(1) (2011) 1-23. http://dx.doi.org/10.1016/j.fss.2011.05.011

[4] L. Frappat, A. Sciarrino and P. Sorba, Crystalizing the genetic code, J. Biological Physics, 27 (2001), 1-34. http://dx.doi.org/10.1023/a:1011874407742

[5] M. K. Kinyon and A. A. Sagle, Quadratic dynamical systems and algebras, J. Differential Equations, 117 (1995), 67-126. http://dx.doi.org/10.1006/jdeq.1995.1049

[6] L. Z. Liu and X. Y. Zhang, Implicative and positive implicative prefilters of EQ-algebras, J. Intell. Fuzzy Systems 26 (2014) 2087-2097.

[7] V. Novák, EQ-algebra-based fuzzy type theory and its extensions, Log. J. IGPL 19 (2011) 512-542. http://dx.doi.org/10.1093/jigpal/jzp087

[8] V. Novák, EQ-algebras: primary concepts and properties, in: Proc. Czech-Japan Seminar, Ninth Meeting, Kitakyushu \& Nagasaki, August 18-22, 2006, Graduate School of Information, Waseda University, 2006, pp. 219-223. 
[9] V. Novák and B. De Baets, EQ-algebras, Fuzzy Sets and Systems 160 (2009) 2956-2978. http://dx.doi.org/10.1016/j.fss.2009.04.010

[10] V. Novák and M. Dyba, Non-commutative EQ-logics and their extensions, in: Proc. World Congress IFSA-EUSFLAT 2009, University of Lisbon, Portugal, 2009.

[11] Rosa M. Rodriguez, Luis Martinez and Francisco Herrera, Hesitant fuzzy linguistic term sets for decision making, IEEE Trans. Fuzzy Syst. 20(1) (2012) 109-119. http://dx.doi.org/10.1109/tfuzz.2011.2170076

[12] R. Sáanchez, R. Grau and E. Morgado, A novel Lie algebra of the genetic code over the Galois field of four DNA bases, Mathematical Biosciences, 202 (2006), 156-174. http://dx.doi.org/10.1016/j.mbs.2006.03.017

[13] J. J. Tian, Evolution Algebras and their Applications, Springer-Verlag, Berlin, Heidelberg, 2008.

[14] J. J. Tian and B. L. Li, Coalgebraic structure of genetics inheritance, Mathematical Biosciences and Engineering, 1 (2004), 243-266. http://dx.doi.org/10.3934/mbe.2004.1.243

[15] V. Torra, Hesitant fuzzy sets, Int. J. Intell. Syst. 25 (2010), 529-539. http://dx.doi.org/10.1002/int.20418

[16] V. Torra and Y. Narukawa, On hesitant fuzzy sets and decision, in: The 18th IEEE International Conference on Fuzzy Systems, Jeju Island, Korea, 2009, pp. 1378-1382. http://dx.doi.org/10.1109/fuzzy.2009.5276884

[17] F. Q. Wang, X. Li and X. H. Chen, Hesitant fuzzy soft set and its applications in multicriteria decision making, J. Appl. Math. Volume 2014, Article ID 643785, 10 pages. http://dx.doi.org/10.1155/2014/643785

[18] G. Wei, Hesitant fuzzy prioritized operators and their application to multiple attribute decision making, Knowledge-Based Systems 31 (2012) 176182. http://dx.doi.org/10.1016/j.knosys.2012.03.011

[19] M. Xia and Z. S. Xu, Hesitant fuzzy information aggregation in decision making, Internat. J. Approx. Reason. 52(3) (2011) 395-407. http://dx.doi.org/10.1016/j.ijar.2010.09.002

[20] M. Xia, Z. S. Xu and N. Chen, Some hesitant fuzzy aggregation operators with their application in group decision making, Group Decision Negotiation 22 (2013) 259-279. http://dx.doi.org/10.1007/s10726-011-9261-7 
[21] Z. S. Xu and M. Xia, Distance and similarity measures for hesitant fuzzy sets, Inform. Sci. 181(11) (2011) 2128-2138. http://dx.doi.org/10.1016/j.ins.2011.01.028

[22] B. Zhu, Z. Xu and M. Xia, Hesitant fuzzy geometric Bonferroni means, Inform. Sci. 205 (2012) 72-85. http://dx.doi.org/10.1016/j.ins.2012.01.048

Received: December 1, 2014; Published: January 7, 2015 